\title{
Ex vivo anticoagulants affect human blood platelet biomechanics with implications for high-throughput functional mechanophenotyping.
}

Laura Sachs ${ }^{1}$, Jan Wesche ${ }^{1}$, Lea Lenkeit ${ }^{1}$, Andreas Greinacher ${ }^{1}$, Markus Bender ${ }^{2}$, Oliver Otto $^{3,4^{*}}$, Raghavendra Palankar ${ }^{*}$

1 Institute for Immunology and Transfusion Medicine, University Medicine Greifswald, Fleischmannstr.8, 17475 Greifswald, Germany

${ }^{2}$ Institute of Experimental Biomedicine - Chair I, University Hospital and Rudolf Virchow Center, Würzburg, Germany.

${ }^{3}$ Zentrum für Innovationskompetenz - Humorale Immunreaktionen bei Kardiovaskulären Erkrankungen, Universität Greifswald, Fleischmannstr. 42, 17489 Greifswald, Germany 4 Deutsches Zentrum für Herz-Kreislauf-Forschung e.V., Standort Greifswald, Universitätsmedizin Greifswald, Fleischmannstr. 42, 17489 Greifswald, Germany.

*corresponding authors

oliver.otto@uni-greifswald.de

raghavendra.palankar@med.uni-greifswald.de 


\begin{abstract}
1 Inherited platelet disorders affecting the human platelet cytoskeleton result in increased

2 bleeding risk. However, deciphering their impact on cytoskeleton-dependent intrinsic

3 biomechanics of platelets remains challenging and represents an unmet need from a

4 diagnostic and prognostic perspective. It is currently unclear whether ex vivo anticoagulants

5 used for the collection of peripheral blood impact the mechanophenotype of cellular

6 components of blood. Using unbiased, high-throughput functional mechanophenotyping of

7 single human platelets by deformability cytometry, we found that ex vivo anticoagulants are a

8 critical pre-analytical variable that differentially influences platelet deformation, their size and

9 functional response to agonists by altering the cytoskeleton. We applied our findings to

10 characterize the functional mechanophenotype of platelets from a patient with Myosin Heavy

11 Chain 9 (MYH9) related macrothrombocytopenia. Our data suggest that platelets from MYH9

12 p.E1841K mutation in humans affecting platelet non-muscle myosin heavy chain IIa

13 (NMMHC-IIA) are biomechanically less deformable in comparison to platelets from healthy

14 individuals.
\end{abstract}




\section{Introduction}

1 Blood platelets are anucleate, discoidal multifunctional cellular fragments (1-3 $\mu \mathrm{m}$ in

2 diameter) generated by bone marrow megakaryocytes and released into blood circulation ${ }^{1}$.

3 On exposed extracellular matrix at the sites of the vascular breach, rapid recruitment of

4 platelets is essential for forming a primary hemostatic plug. However, under pathological

5 procoagulatory conditions, platelets contribute to intravascular thrombosis, a leading cause of

6 cardiovascular complications and morbidities ${ }^{2-4}$. Platelets function as complex biological

7 sensor and actuator units that respond to a broad spectrum of physicochemical stimuli via

8 ligand-receptor-mediated interactions (i.e., outside-in signaling) and mechanotransduction

9 events (i.e., both outside-in and inside-out signaling) ${ }^{5-7}$. This complex interplay results in the

10 coordinated regulation of signaling kinetics, including cytoskeletal remodeling that initiates

11 platelet adhesion, activation, spreading, and platelet contraction ${ }^{8}$.

12 It has been well established that cytoskeleton-dependent biomechanics governs diverse

13 aspects of platelet function during hemostasis and thrombosis ${ }^{9,10}$. Beyond this, the

14 significance of platelet cytoskeletal integrity and its functional role in platelet-mediated innate

15 immune responses such as mechano-scavenging, host defense during platelet-bacteria

16 interactions, and vascular surveillance is emerging ${ }^{11-13}$. Recent studies have also

17 demonstrated changes in platelet biomechanical properties, and subsequent defective

18 mechanotransduction may serve as a biophysical marker for assessing bleeding risk in

19 individuals with inherited platelet cytoskeletal defects ${ }^{14}$. Thus, deciphering cytoskeleton-

20 dependent intrinsic biomechanical properties of platelets is highly relevant not only for

21 broadening our understanding of the functional role of platelets in physiological and

22 pathological processes but also from translationally significant diagnostic and prognostic

23 perspectives ${ }^{6,10}$.

24 Currently, a wide array of biophysical methods is available for the investigation of platelet

25 biomechanics. They include micropipette aspiration ${ }^{15-17}$, atomic force microscopy ${ }^{18-20}$, 
1 scanning ion conductance microscopy ${ }^{21,22}$, traction force microscopy ${ }^{23,24}$, including flexible

2 micropost arrays ${ }^{25-27}$. Although these methods have proven valuable in advancing our

3 insights into platelet biomechanics, these are technically demanding, labor-intensive, and

4 mostly limited to analysis of adherent platelets ${ }^{28}$. Besides, these methods also lack

5 throughput, which results in implicit bias during single platelet measurements resulting from

6 under-sampling of innate heterogeneity found in donor platelet populations ${ }^{29-31}$.

7 The recently introduced on-chip, high-throughput real-time fluorescence and deformability 8 cytometry (RT-FDC) has rapidly emerged as a biophysical method to address these

9 challenges ${ }^{32,33}$. RT-FDC enables continuous on-the-fly mechanophenotyping of single cells

10 at real-time analysis rates exceeding 1000 cells/s combined with the capability of achieving

11 molecular specificity through the application of fluorescent probes, which further opens up

12 exciting possibilities ${ }^{34-37}$.

13 However, on-chip deformability cytometry and other biophysical methods have not been well

14 standardized regarding pre-analytical variability in sample preparation of cells from

15 peripheral blood, including platelets ${ }^{38}$. Specifically, it is unclear whether different ex vivo 16 anticoagulants commonly used during blood sampling influence blood platelet biomechanics.

17 Using high-throughput functional mechanophenotyping of single platelets in RT-FDC, here we demonstrate that ex vivo anticoagulants differentially impact intrinsic biomechanical properties (i.e., deformation and size) of human platelets. Besides this, we establish a link between platelet functional mechanophenotype, particularly their deformation and associated activation profiles as well as functional response in resting platelets and after activation with platelet agonist, respectively, in different ex vivo anticoagulants. We explain these findings by showing that ex vivo anticoagulants and platelet activation alter the content and subcellular organization of major platelet cytoskeletal components such as actin cytoskeleton and marginal band tubulin ring. Furthermore, in a potentially diagnostically significant 
1 platelet cytoskeletal disorder, affecting platelet non-muscle myosin heavy chain IIa

2 (NMMHC-IIA), we demonstrate that the choice of ex vivo anticoagulant may strongly impact

3 the outcomes of mechanophenotyping.

\section{Results}

Ex vivo anticoagulants affect human platelet deformation and size.

4 We first evaluated the effects of ex vivo anticoagulants on platelet deformation, and their

5 corresponding size in live non-stimulated (i.e., resting) platelets in PRP by RT-FDC prepared

6 from blood collected in ACD-A, Na-Citrate, K2-EDTA, Li-Heparin, and r-Hirudin (Fig. 1a).

7 Non-stimulated platelets showed deformation of $0.127 \pm 0.033$ (mean $\pm S D, n=6$ donors) in

8 ACD-A, $0.111 \pm 0.025$ in Na-Citrate, and $0.1 \pm 0.023$ in r-Hirudin, which was significantly

9 higher in comparison to the less deformable platelets at $0.071 \pm 0.016$ in Li-Heparin and

$100.037 \pm 0.01$ in $\mathrm{K}_{2}$-EDTA. (Fig. $1 \mathrm{~b}$ and Supplementary Fig. 4 for statistical distribution plots

11 of individual donors ). Assessment of corresponding platelet size in different ex vivo 12 anticoagulants from non-stimulated platelets revealed differences in platelet size measuring at $135.035 \pm 0.49 \mu \mathrm{m} 2$ (mean $\pm \mathrm{SD}, \mathrm{n}=6$ donors) in ACD-A in comparison to K2-EDTA and Li14 Heparin where platelets were significantly smaller in size measuring at $4.158 \pm 0.241 \mu \mathrm{m} 2$ 15 and $4.337 \pm 0.344 \mu \mathrm{m} 2$, respectively (Fig. 1c and Supplementary Fig. 5 for statistical 16 distribution plots of individual donors). 
a. Non-stimulated (resting platelets)
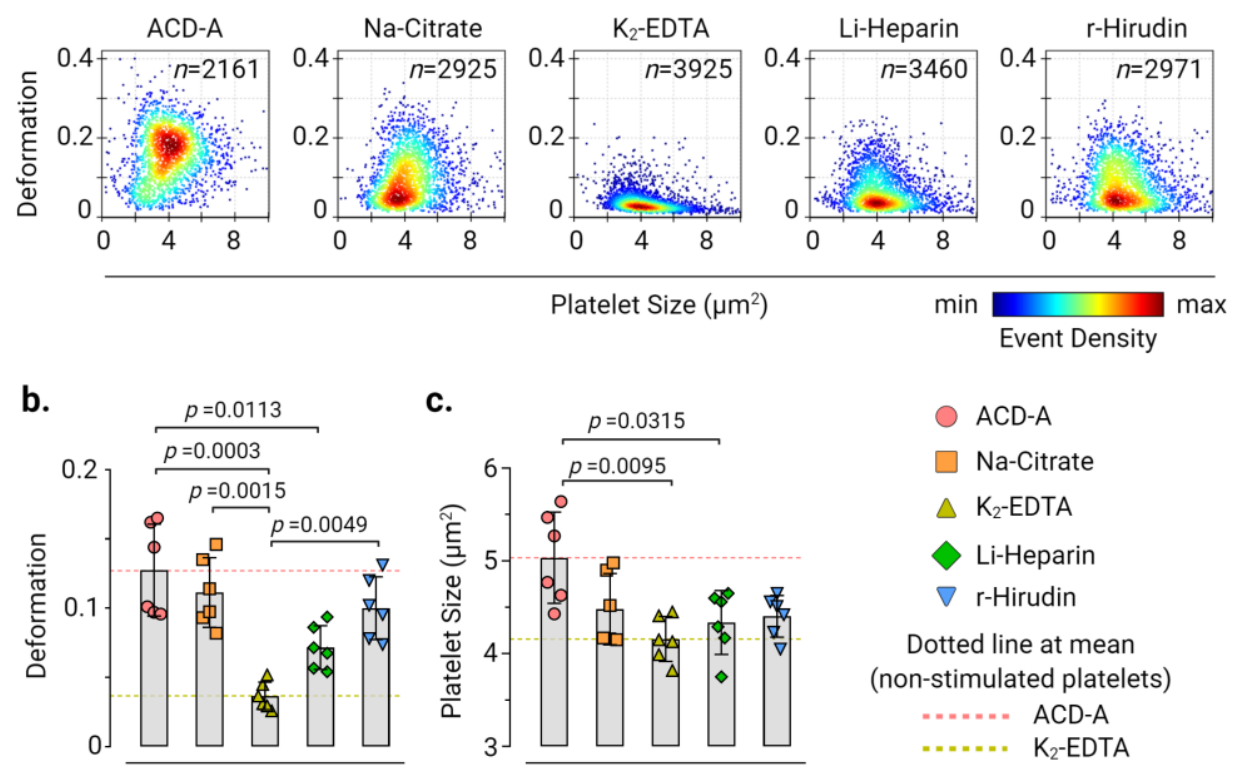

d. TRAP-6 stimulated

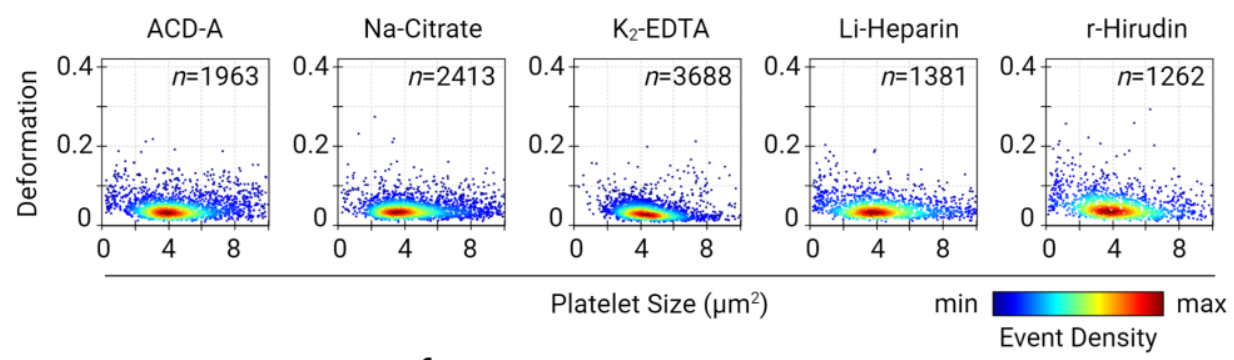

e.

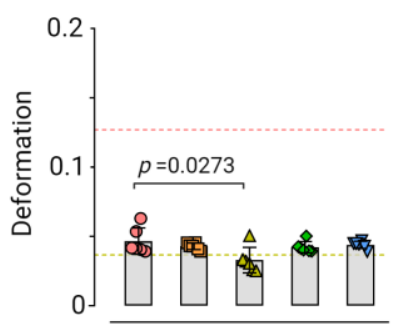

g.

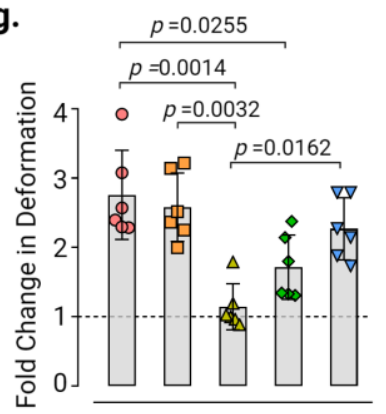

f.

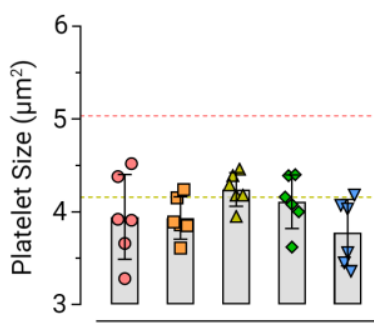

h.

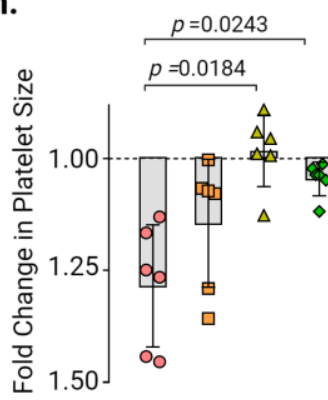

ACD-A

$\square$ Na-Citrate

$\triangle \mathrm{K}_{2}$-EDTA

Li-Heparin

$\nabla$ r-Hirudin

Dotted line at mean (non-stimulated platelets)

...... ACD-A

$\ldots K_{2}$ EDTA

Fig 1: Ex vivo anticoagulants influence the deformability and size of human platelets. Representative KDE scatter plots of deformation and size of live single platelets in PRP prepared from whole blood collected in different ex vivo anticoagulants from (a) nonstimulated (i.e., resting) platelets and (d) after stimulation with TRAP-6 ( $n=$ number of single 
platelets from the same donor measured for each condition). Color coding of event density in scatter plots indicates a linear density scale from min (blue) to max (dark red). Summary data points show the median values of individual donors, and bar plots show mean \pm S.D. of platelet deformation and size from non-stimulated (b) and (c) and TRAP-6 stimulated platelets (e) and (f), respectively ( $\mathrm{n}=6$ donors). Fold change in platelet deformation and size upon stimulation with TRAP-6 are shown in (g) and (h), respectively, where dotted control baseline $=1$ ( $\mathrm{n}=6$ donors). Statistical analysis: mixed-effects model (restricted maximum likelihood, REML) followed by Tukey's multiple comparisons tests, with single pooled variance and $p>0.05$ was considered significant.

1 Next, to test whether agonist-induced platelet activation leads to measurable changes in

2 platelet deformation and their corresponding size depending on the type of ex vivo 3 anticoagulant, TRAP-6 was used. Platelet activation by TRAP-6 resulted in a noticeable

4 decrease in platelet deformation and a concomitant reduction in platelet size in all ex vivo

5 anticoagulants except for $\mathrm{K}_{2}$-EDTA (Fig. 1d, 1e, and 1f). Assessment of fold-change in 6 platelet deformation before and after TRAP-6 stimulation showed a decrease in platelet 7 deformation by a factor of $2.76 \pm 0.64$ (mean $\pm \mathrm{SD}, \mathrm{n}=6$ donors) in ACD-A, $2.58 \pm 0.49$ in Na-

8 Citrate, $1.72 \pm 0.47 \mathrm{Li}$-Heparin, and $2.27 \pm 0.45$ in r-Hirudin (Fig. 1g). On the contrary, in $\mathrm{K}_{2^{-}}$

9 EDTA, TRAP-6 stimulation resulted in a minimal fold change in platelet deformation by a

10 factor of $1.14 \pm 0.33$ (Fig. 1g). Similarly, platelet size decreased upon TRAP-6 stimulation by

11 a factor of $1.28 \pm 0.13$ (mean $\pm \mathrm{SD}, \mathrm{n}=6$ donors) in ACD-A, $1.14 \pm 0.14$ in Na-Citrate and

$121.18 \pm 0.16$ in $\mathrm{r}$-Hirudin, while it remained unchanged at $0.98 \pm 0.08$ in $\mathrm{K}_{2}$-EDTA and $1.04 \pm$ 0.04 in Heparin (Fig. 1h). Furthermore, the changes in platelet shape observed in the RT-FDC differed between the ex vivo anticoagulants in non-stimulated and TRAP-6 stimulated platelets (Supplementary Fig. 6 representative bright-field images of single platelets in measurement channel overlaid with contour). 


\section{Platelet deformation in response to platelet activation}

1 In non-stimulated platelets, basal CD62P surface expression was not altered between all ex

2 vivo anticoagulants even though platelets in $\mathrm{K}_{2}$-EDTA exhibited decreased deformation

3 relative to other ex vivo anticoagulants (Fig. 2a, 2b, and 2c). Upon activation of platelets by

4 TRAP-6, a significant decrease in platelet deformation with a concomitant increase in CD62P

5 surface expression and CD62P \% positive platelets was observed in all ex vivo anticoagulants

6 except in $\mathrm{K}_{2}$-EDTA (Fig. 2d, 2e, and 2f). Assessment of fold-change in CD62P expression

7 levels showed a significant increase by a factor of $18.19 \pm 8.88$ (mean $\pm \mathrm{SD}, \mathrm{n}=6$ donors) in

8 ACD-A, $21.48 \pm 8.54$ in Na-Citrate, $9.82 \pm 7.78$ in Li-Heparin, and $15.72 \pm 6.76$ in r-Hirudin

9 in comparison to a fold change of $2.03 \pm 1.16$ in $\mathrm{K}_{2}$-EDTA (Fig. $2 \mathrm{~g}$ ). Multivariate analysis of

10 continuous variables from RT-FDC data from non-stimulated and TRAP-6 stimulated

11 platelets (Fig. 2h and 2i) further confirmed platelet deformation, size, and CD62P expression

12 is strongly affected in $\mathrm{K}_{2}$-EDTA. 
a. Non-stimulated (resting platelets)

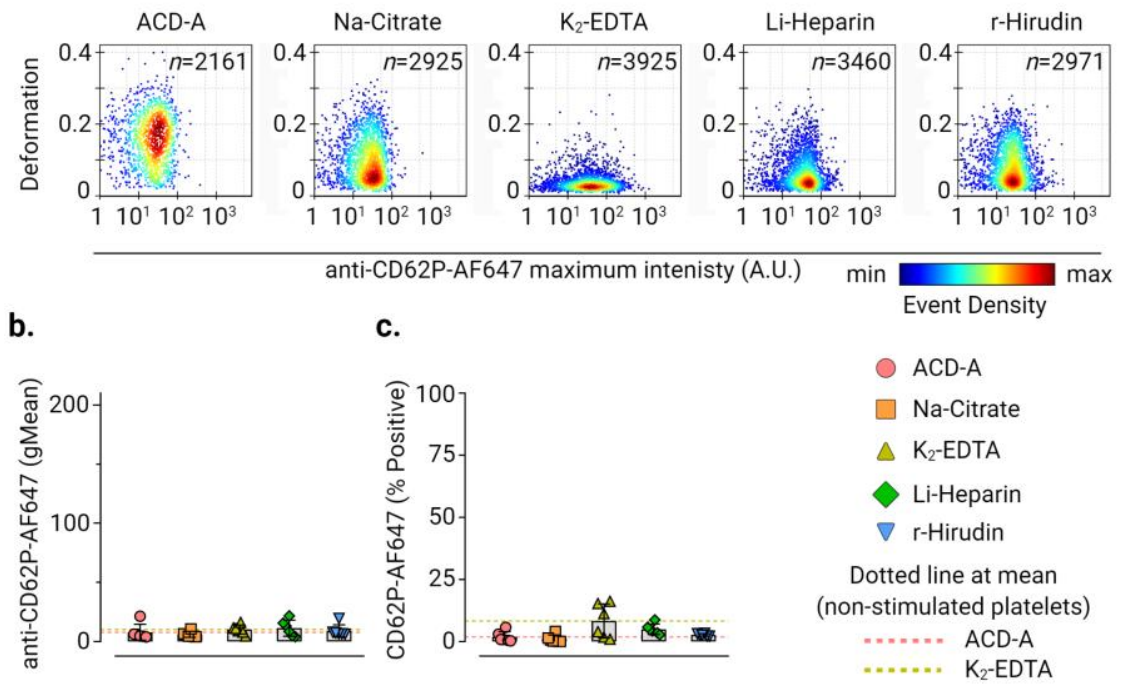

d. TRAP-6 stimulated

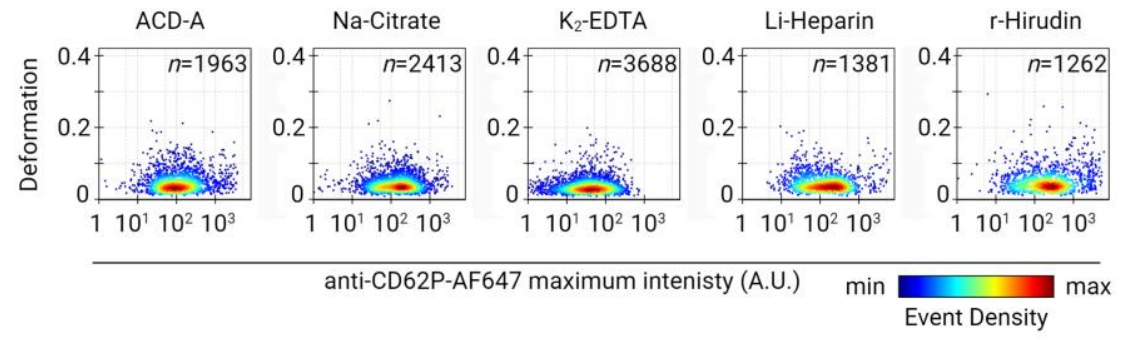

e.

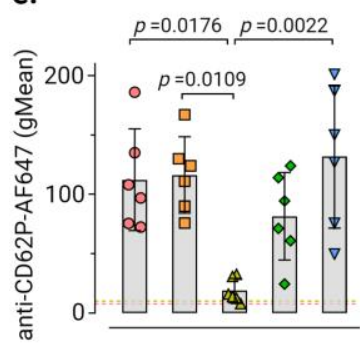

h. Non-stimulated

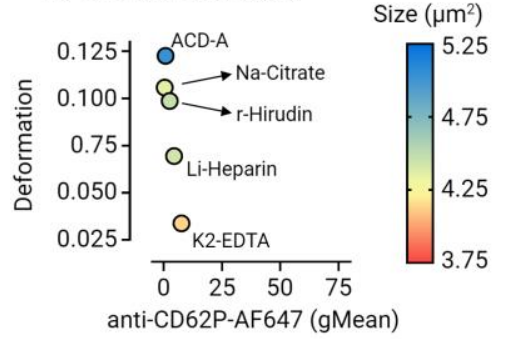

f.

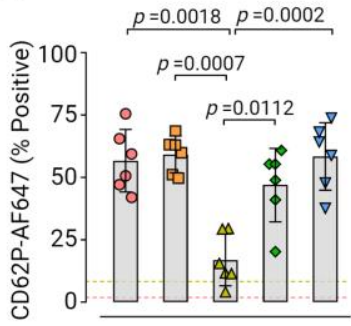

g.

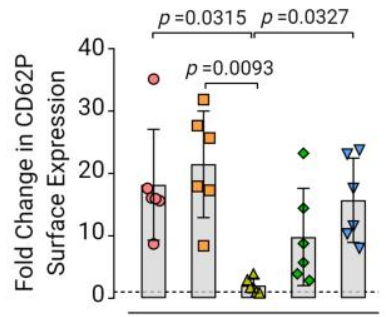

Fig 2: Platelet deformability and the corresponding CD62P surface expression upon activation differs in different $e x$ vivo anticoagulants. Representative KDE scatter plots of platelet deformation and CD62P expression on single platelets expression (plotted on $\log 10$ scale of maximum intensity in arbitrary units (A.U.) of anti-CD62P-AlexaFluor647 antibody) in PRP different ex vivo anticoagulants in (a) non-stimulated (i.e., resting) platelets and (d) upon TRAP-6 stimulation. ( $\mathrm{n}=$ number of single platelets from the same donor measured for each condition ). Color coding of event density in scatter plots indicates a linear density scale 
from min (blue) to max (dark red). Summary graphs show of median values of individual donors, while bar graphs show mean \pm S.D. of geometric mean fluorescence intensity (gMean) of CD62P expressing platelets (b) and (c) and percent positive platelets (e) and (f) above the cut-off of 5000 events or $10 \mathrm{~min}$ in non-stimulated and TRAP-6 stimulated platelets, respectively ( $\mathrm{n}=6$ donors). Fold change in CD62P surface expression on platelets upon stimulation with TRAP-6 are shown in $(\mathbf{g})$ where dotted control baseline $=1(\mathrm{n}=6$ donors). Multivariate analysis plots of continuous variables from RT-FDC, (h) and (i) of nonstimulated and TRAP-6 stimulated platelets, respectively, displaying the relationships between platelet deformability, size, and related CD62P surface expression levels in different ex vivo anticoagulants. (Data represents median values of individual variables from $n=6$ donors). Statistical analysis: mixed-effects model (restricted maximum likelihood, REML) followed by Tukey's multiple comparisons tests, with single pooled variance and $p>0.05$ was considered significant.

1 Next, we assessed conformational changes in platelet integrin $\alpha_{\mathrm{IIb}} \beta_{3}$ as a marker for platelet activation by PAC-1 antibody binding (Fig. 3). Baseline activation levels of integrin $\alpha_{\mathrm{IIb}} \beta_{3}$ in non-stimulated platelets (Fig. 3a, 3b, and 3c) were highest in Li-Heparin (PAC-1-FITC gMean of $82.48 \pm 12.16$ and PAC-1-FITC \% positive platelets at $36.02 \% \pm 9.3$, mean \pm S.D.,

$5 \mathrm{n}=6$ donors). In contrast, K2-EDTA showed the lowest basal activation of integrin $\alpha_{\mathrm{IIb}} \beta_{3}$ of all ex vivo anticoagulants. In TRAP-6 stimulated platelets, PAC-1-FITC binding and PAC-1FITC \% positive platelets increased significantly $(p<0.0001)$ in all ex vivo anticoagulants in comparison to $\mathrm{K}_{2}$-EDTA (Fig. 3d, 3e, and 3f). Furthermore, fold change in PAC-1 binding to platelets after TRAP-6 stimulation increased by a factor of $2.86 \pm 0.82$ (mean $\pm \mathrm{SD}, \mathrm{n}=6$ donors) in ACD-A, $3.39 \pm 0.9$ in Na-Citrate, $2.64 \pm 0.7$ in Li-Heparin, and $3.55 \pm 0.98$ in rHirudin in comparison to non-stimulated platelets, but did not increase in $\mathrm{K}_{2}$-EDTA (Fig. $3 \mathrm{~g}$ ).

12 Also, multivariate analysis of continuous variables from RT-FDC data from non-stimulated and TRAP-6 stimulated platelets (Fig. 3h and 3i) revealed together with platelet deformation, size, and PAC-1 binding is strongly affected in $\mathrm{K}_{2}$-EDTA. Our results concerning the reduced binding of PAC-1 antibody to platelet integrin $\alpha \mathrm{IIb} \beta 3$ are consistent with previous observations in potent chelators of divalent cations such as EDTA ${ }^{39}$. 
a. Non-stimulated (resting platelets)

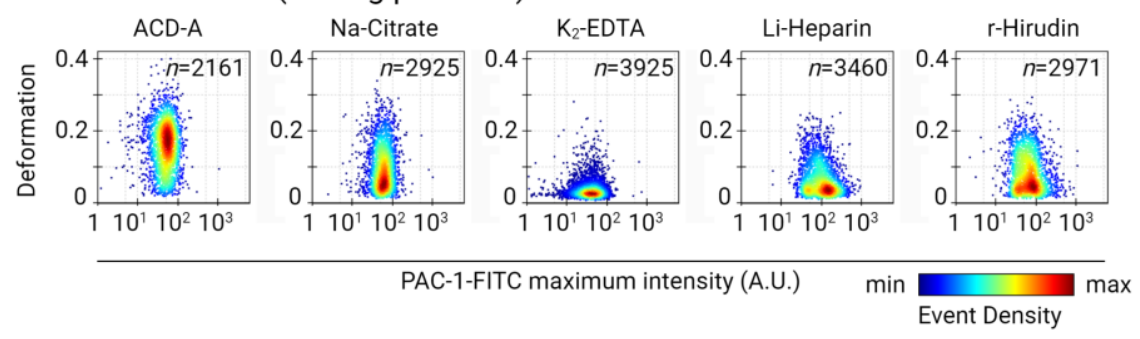

b.

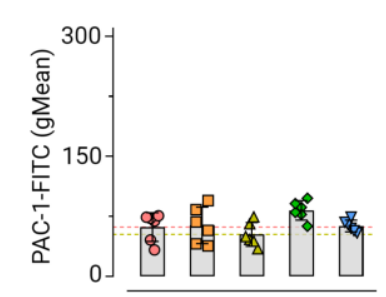

c.

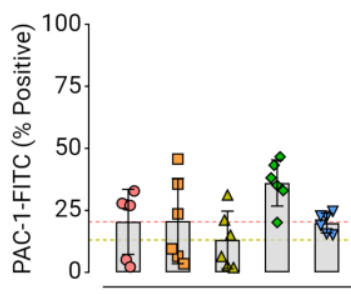

ACD-A

$\square$ Na-Citrate

$\triangle \mathrm{K}_{2}$-EDTA

$\checkmark$ Li-Heparin

$\nabla$ r-Hirudin

Dotted line at mean (non-stimulated platelets)

..... ACD-A

....... K K K$_{2}$ EDTA

d. TRAP-6 stimulated

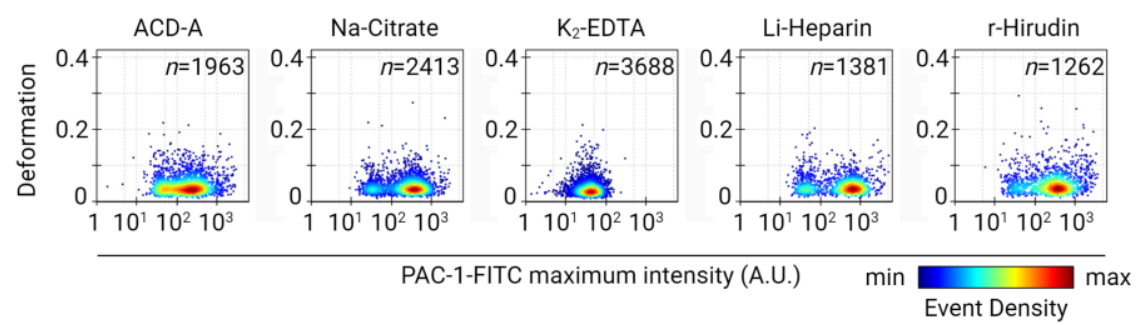

e.

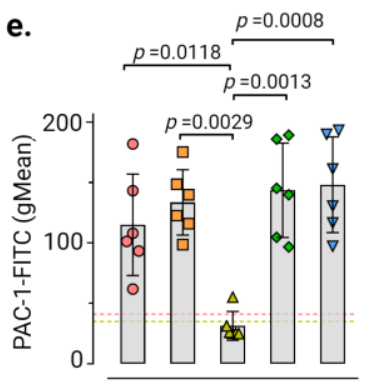

h. Non-stimulated

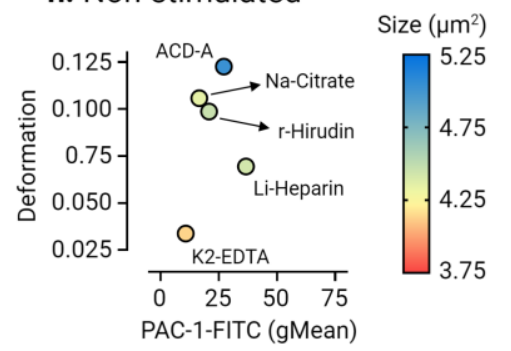

g. $\quad p=0.0157 \stackrel{p=0.0003}{p}$

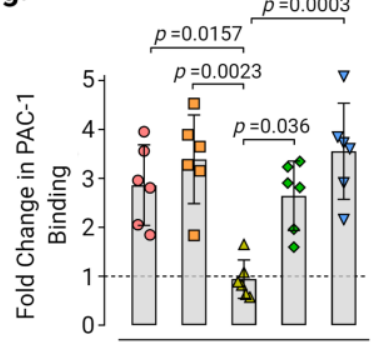

i. TRAP-6 stimulated

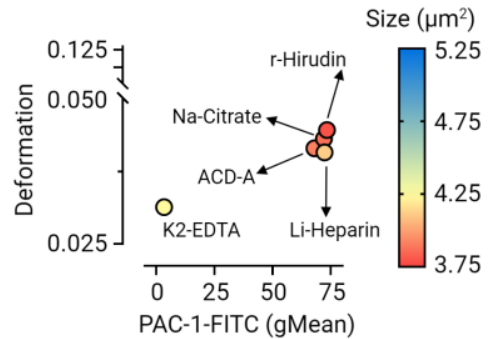

Fig 3: Platelet deformation and activation-induced exposure of the conformational epitope of the integrin $\alpha \mathrm{IIb \beta 3}$ is strongly influenced by ex vivo anticoagulants. Representative KDE scatter plots of platelet deformation and their corresponding activation status as a readout for binding of integrin $\alpha \operatorname{IIb} \beta 3$ specific ligand-mimetic PAC- 1 antibody (plotted on $\log 10$ scale of maximum intensity in arbitrary units (A.U.) of PAC-1-FITC antibody) on single platelets in different ex vivo anticoagulants in (a) non-stimulated (i.e., resting) platelets and upon stimulation TRAP-6 (b) from a single donor ( $\mathrm{n}=$ number of single platelets from the same donor measured for each condition). Color coding of event density in 
scatter plots indicates a linear density scale from min (blue) to max (dark red). Summary graphs show of median values of individual donors, while bar graphs show mean \pm S.D. of geometric mean fluorescence intensity (gMean) of PAC-1-FITC antibody bound to platelets (c) and (d) and PAC-1-FITC antibody percent positive platelets (e) and (f) above the cut-off of 5000 events or $10 \mathrm{~min}$ in non-stimulated and TRAP-6 stimulated platelets, respectively $(\mathrm{n}=$ 6 donors). Fold change in CD62P surface expression on platelets upon stimulation with TRAP-6 from six donors are shown in (g) where dotted control baseline $=1$ ( $n=6$ donors). Multivariate analysis plots of continuous variables from RT-FDC, (h) and (i) of nonstimulated and TRAP-6 stimulated platelets, respectively, displaying the relationships between platelet deformability, size, and PAC-1 antibody biding to integrin $\alpha \operatorname{IIb} \beta 3$ in different ex vivo anticoagulants. (Data represents median values of individual variables from $\mathrm{n}=6$ donors). Statistical analysis: mixed-effects model (restricted maximum likelihood, REML) followed by Tukey's multiple comparisons tests, with single pooled variance and $\mathrm{p}$ > 0.05 was considered significant.

\section{Increased platelet stiffness is an indicator of alterations in platelet cytoskeletal} organizations and F-actin content.

1 Fluorescence CLSM imaging and subsequent line profile analysis of non-stimulated platelets

2 in ACD-A, Na-Citrate, Li-Heparin, and r-Hirudin showed discoidal morphology, a uniform 3 intracellular distribution of F-actin (Phalloidin, green), and a well-defined sub-cortical

4 marginal band microtubule ring ( $\alpha$-tubulin, edge-to-edge fluorescence intensity signal in 5 magenta) (Fig. 4a and 4b). In contrast, platelets in $\mathrm{K}_{2}$-EDTA platelets lost their discoidal 6 shape and were comparatively smaller. Besides, we observed an increase in subcortical

7 localization of F-actin and coiling of microtubule ring (indicated by white arrowhead in 8 grayscale sub-figures in Fig 4a and edge-to-edge fluorescence line profile intensity in $4 \mathrm{~b}$ for $9 \mathrm{~K}_{2}$-EDTA). Upon TRAP-6 stimulation, platelets showed substantial morphological changes compared to their non-stimulated counterparts (Fig. 4c). Line profile analysis of fluorescence

11 intensities further revealed an increased F-actin localization at sub-cortical regions and a

12 decrease in edge-to-edge coiling of microtubule ring in all ex vivo anticoagulants except for $\mathrm{K}_{2}$-EDTA (Fig.4d). 
a. Non-stimulated (resting platelets)
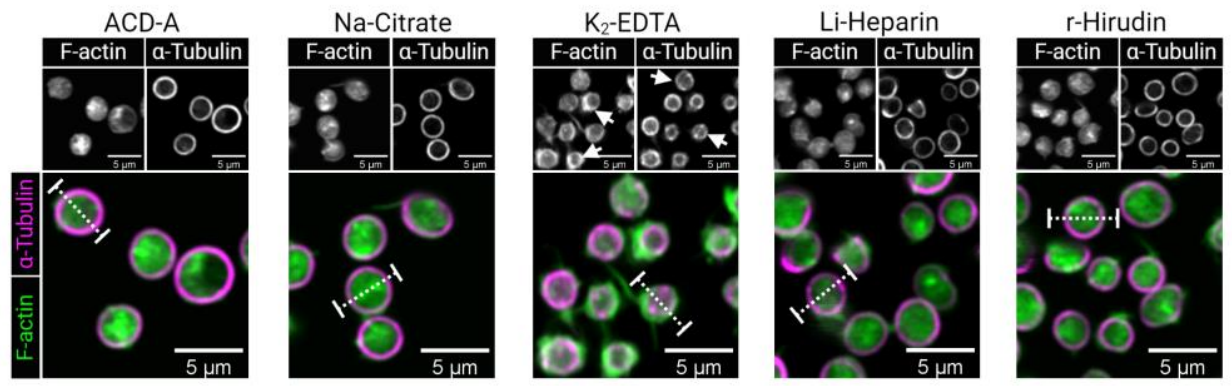

b.
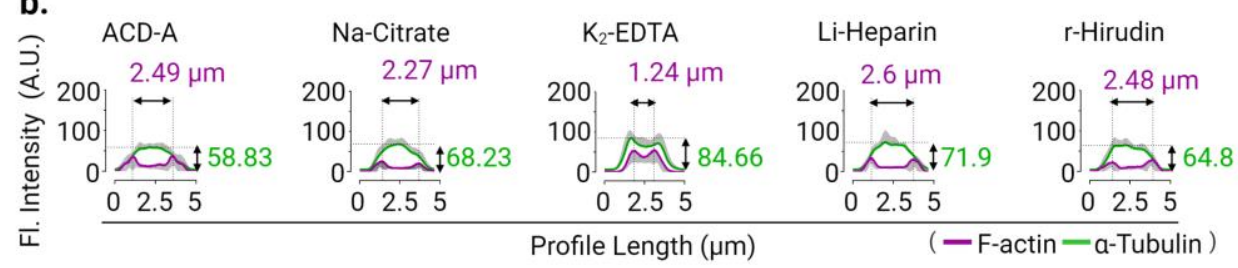

c. TRAP-6 stimulated
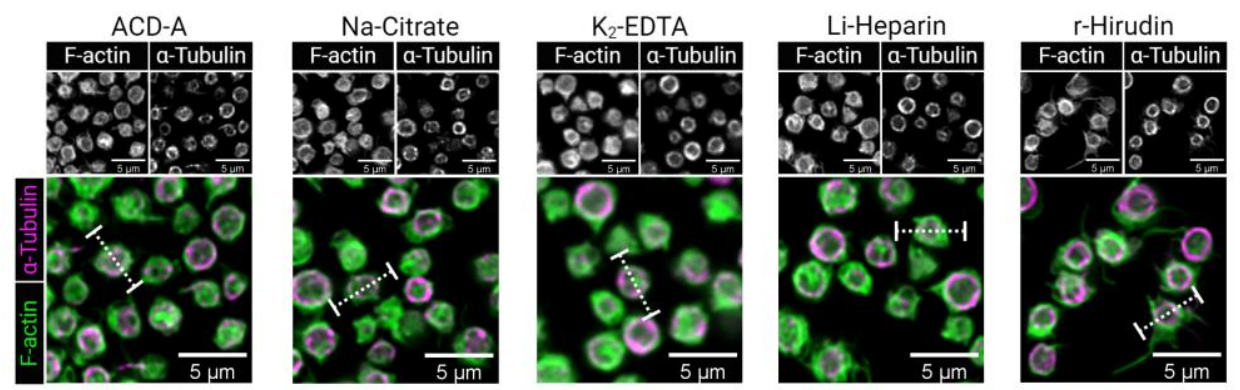

d.
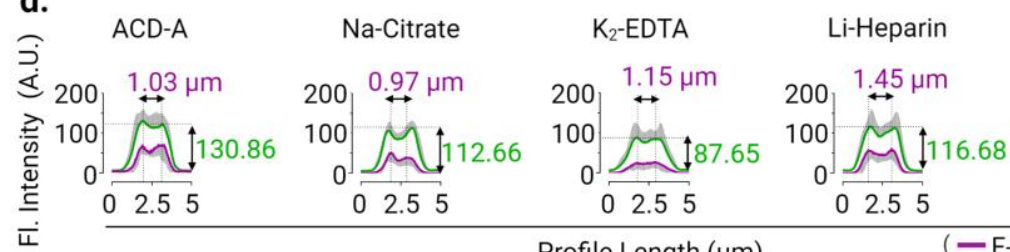

r-Hirudin

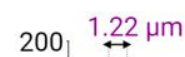

Profile Length $(\mu \mathrm{m})$

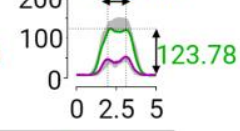

Profle Length $($ m)

Fig 4: Cytoskeletal organization in resting and TRAP-6 stimulated human platelets are altered in different $e x$ vivo anticoagulants. Representative confocal laser scanning fluorescence microscopic images of F-actin (green) distribution and marginal band $\alpha$-tubulin (magenta) organization of human platelets in different ex vivo anticoagulants under (a) nonstimulated (resting platelets) and (c) 10 minutes after TRAP-6 stimulation. Fluorescence intensity line profiles (represented by the white dotted line) across individual platelets (b) and (d) show fluorescence intensity (A.U.) (along Y-axis for F-actin in green), and immunofluorescence detection of circumferential marginal band $\alpha$-tubulin ring (magenta) shown as a measure of the change in the edge-to-edge length $(\mu \mathrm{m})($ plotted along X-axis in magenta). Graphical plots show mean \pm S.D. from fluorescence intensity (A.U.) of $n=10$ single platelets per ex vivo anticoagulant. 
1 Next, we analyzed the total F-actin content in non-stimulated and TRAP-6 stimulated

2 platelets in different ex vivo anticoagulants by flow cytometry (Fig.5a and $5 \mathrm{~b}$ and

3 Supplementary Fig.7). We observed a significantly higher F-actin content in non-stimulated

4 platelets in $\mathrm{K}_{2}$-EDTA (Phalloidin AF647 fluorescence gMean: $162.2 \pm 30.47$, mean \pm CD

5 from $\mathrm{n}=6$ donors) in comparison to non-stimulated platelets measuring at $110.7 \pm 32.11$,

$6 p=0.036$, in ACD-A, $107 \pm 23.86, p=0.0285$ in Na-Citrate and $94.24 \pm 28.91, p=0.0023$ in r-

7 Hirudin (Fig. 5a). The basal F-actin content of platelets in Li-Heparin was found to be 8 relatively higher (Phalloidin AF647 fluorescence gMean: $146.5 \pm 9$, mean \pm CD from $n=6$

9 donors) than in ACD-A and Na-Citrate, statistically significant differences $(p=0.024)$ were

10 apparent between Li-Heparin and r-Hirudin in non-stimulated platelets (Fig. 5a). TRAP-6

11 stimulation resulted in a significant increase of total F-actin in platelets by a factor of $2.18 \pm$

120.28 in ACD-A, $2.13 \pm 0.38$ in Na-Citrate, and $2.1 \pm 0.27$ in r-Hirudin. In contrast, only a

minor change in total F-actin content by a factor of $1.49 \pm 0.26$ in Li-Heparin and $0.96 \pm 0.14$

in $\mathrm{K}_{2}$-EDTA was observed (Fig. 5b, 5c and Supplementary Fig.7).

Next, a multivariate analysis of continuous variables was performed to verify whether the changes in actin polymerization status, i.e., total F-actin content measured by flow cytometry, reflect the observed differences in platelet deformation and their corresponding size by RTFDC in different ex vivo anticoagulants. As shown, non-stimulated (i.e., resting) platelets Hirudin than those in Li-Heparin. Furthermore, platelets in $\mathrm{K}_{2}$-EDTA deformed least with higher basal F-actin content and smallest in size. Under TRAP-6 stimulation, except in $\mathrm{K}_{2^{-}}$ EDTA, platelets in all ex vivo anticoagulants showed decreased deformation, a smaller size, and increased total F-actin content (Fig 5e). 
a. Non-stimulated

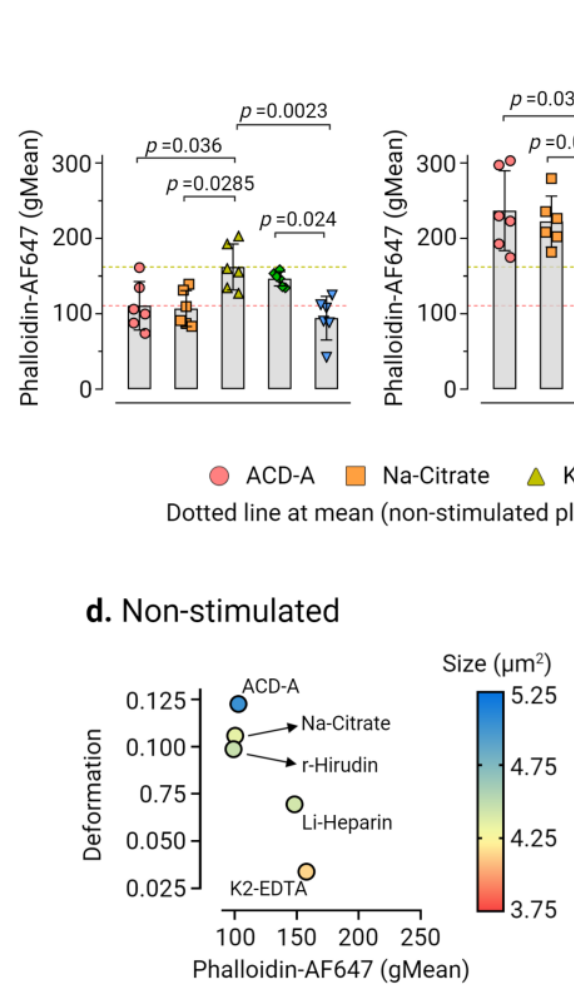

c. Fold change in F-actin content

$$
p<0.0001 p<0.0001
$$

$p=0.0077$

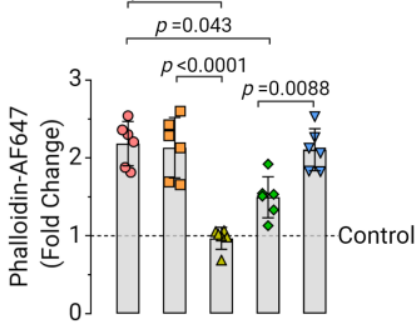

0 


\section{ACD-A, but not K2-EDTA, allows mechanophenotyping of MYH9 related disease mutations in human platelets}

1 By RT-FDC, we next analyzed platelets from an individual with $M Y H 9$ p.E1841K mutation in

2 the rod region of NMMHC-IIA, an essential platelet cytoskeletal protein ${ }^{40}$. In ACD-A, MYH9

3 p.E1841K platelets in comparison to platelets from healthy controls deform less $(0.068$, 4 median $\mathrm{n}=955$ single platelets vs. 0.122 , median $\mathrm{n}=2326$ single platelets) and larger (5.77 $5 \mu \mathrm{m}^{2}$, median $\mathrm{n}=955$ single platelets vs. $4.05 \mu \mathrm{m}^{2}$, median $\mathrm{n}=2326$ single platelets) (Fig. $6 \mathrm{a}$ and 6 Supplementary Fig.8a) under non-stimulated (i.e. resting) conditions. With TRAP-6 7 stimulation, platelets from the individual with $M Y H 9$ p.E1841K in ACD-A deform further 8 less $(0.036$, median $\mathrm{n}=1112$ single platelets $)$, but intriguingly their size increased $\left(6.585 \mu \mathrm{m}^{2}\right.$, 9 median $\mathrm{n}=1112$ single platelets). In contrast, the platelets from the healthy individual showed 10 decreased deformation (0.0455, median $n=720$ single platelets) and size $\left(2.595 \mu \mathrm{m}^{2}\right)($ Fig. $6 \mathrm{~b}$ 11 and Supplementary Fig.8b). On the other hand, in $\mathrm{K}_{2}$-EDTA, non-stimulated healthy control 12 platelets showed $\approx 3$ fold decreased deformation ( 0.036 , median $\mathrm{n}=1960$ single platelets) and while $M Y H 9$ p.E1841K platelets showed a $\geq 3$ fold decreased deformation (0.0195, median $\mathrm{n}=2406$ single platelets) in comparison to their counterparts in ACD-A (Fig. 6c and Supplementary Fig.9a).

16 Besides, in $\mathrm{K}_{2}$-EDTA, platelets from the healthy individual became smaller $\left(3.27 \mu \mathrm{m}^{2}\right.$, median $\mathrm{n}=1960$ single platelets). In contrast, platelets from the MYH9 p.E1841K individual were slightly increased in their size $\left(7.515 \mu \mathrm{m}^{2}\right.$, median $\mathrm{n}=2406$ single platelets $)$ compared to nonstimulated platelets in ACD-A. Furthermore, TRAP-6 stimulation of platelets in K2-EDTA only resulted in minor changes in platelet deformation and size compared to their nonstimulated counterparts (Fig. 6d and Supplementary Fig.9b). Although differences in platelet deformability and size were apparent between the platelets from $M Y H 9$ p.E1841K patient and healthy control in ACD-A; their CD62P surface expression levels and PAC-1 binding in response to TRAP-6 were comparable. 
a. ACD-A (non-stimulated)

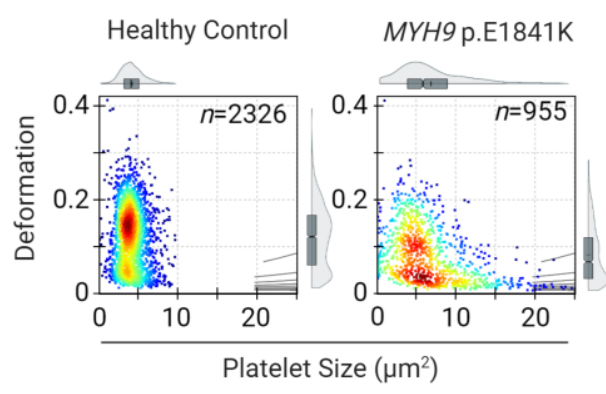

C. K2-EDTA (non-stimulated)

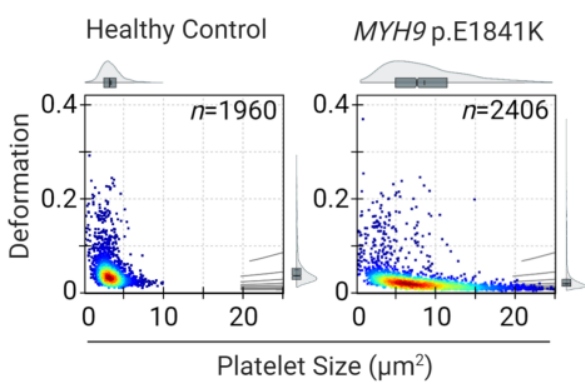

b. ACD-A (TRAP-6 stimulated)

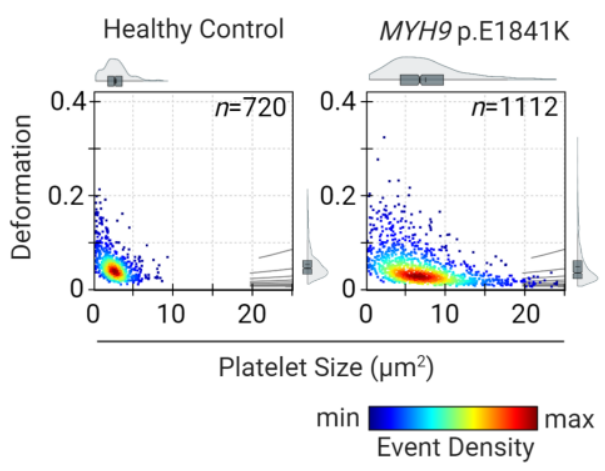

d. K2-EDTA (TRAP-6 stimulated)

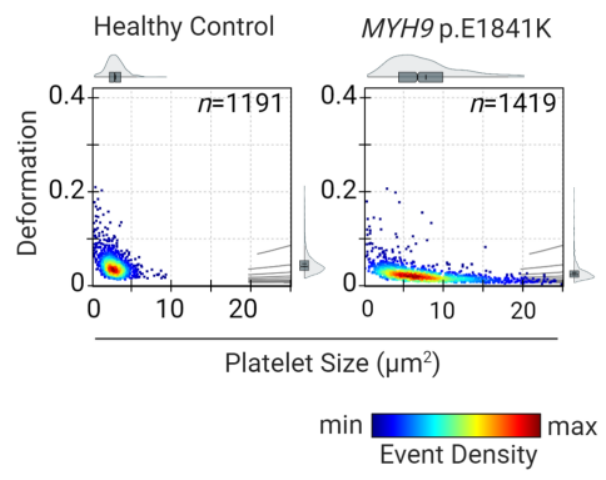

Fig 6: Deformability and size of platelets from a healthy individual and from a patient carrying MYH9 p.E1841K mutation. KDE scatter plots from RT-FDC measurements performed on the same day of displaying the distribution of single platelet deformability and their corresponding size between single platelets from a healthy individual (control) and from a patient carrying the mutation $M Y H 9$ p.E1841K for non-muscle myosin heavy chain IIa, in ex vivo anticoagulant (a) ACD-A and (b) in $\mathrm{K}_{2}$-EDTA before and after stimulation with platelet agonist TRAP-6, (c) and (d), respectively. ( $\mathrm{n}=$ number of single platelets). Statistical distribution plots for each condition: a notch in box plot and the horizontal line depicts median and mean, respectively, and the interquartile ranges. The full distribution of the data for each parameter is depicted by violin plots.

1 Consistent with our observations reported above, in K2-EDTA platelets from both MYH9

2 p.E1841K patient and healthy control failed to respond to TRAP-6 (Supplementary Fig. 10

3 and Fig. 11). Furthermore, in ACD-A, assessment of F-actin content revealed a higher basal

4 total F-actin content in non-stimulated platelets from $M Y H 9$ p.E1841K patient at 192.86

5 (Phalloidin AF647 gMean) compared to the healthy control at 154.96, which upon TRAP-6

6 stimulation increased to 250.16 and 286.36, respectively, (Supplementary Fig.12a). On the 
1 other hand, in $\mathrm{K}_{2}$-EDTA, the basal total F-actin content in non-stimulated platelets from

2 MYH9 p.E1841K patient was found to be at 250.42 (Phalloidin AF647 gMean) and for

3 healthy control at 206.6, that remained unchanged upon TRAP-6 stimulation (Supplementary $4 \quad$ Fig.12b). 


\section{Discussion}

1 The present study shows that $\mathrm{K}_{2}$-EDTA and Li-Heparin should not be used as ex vivo

2 anticoagulants for studies on human platelet biomechanical properties. Platelets collected in

3 ACD-A, Na-Citrate, or r-Hirudin may be used for biomechanical studies. Still, due to minor

4 differences in the effects on platelets, results cannot be directly compared between platelets

5 anticoagulated with these different anticoagulants. Platelets anticoagulated with Li-Heparin

6 show some differences in their biomechanical characteristics compared to platelets in ACD-

7 A, Na-Citrate, or r-Hirudin. Heparinized platelets show a twofold higher F-actin content,

8 decreased deformation, and higher PAC-1 expression. The most crucial difference between

9 Li-Heparin and r-Hirudin compared to the other anticoagulants is that Li-Heparin and r-

10 Hirudin do not chelate calcium. However, the apparent discrepancies between Li-Heparin and

11 r-Heparin indicate that Li-Heparin is inducing artifacts in the biomechanical properties of platelets. One explanation is the strong negative charge of heparin and its binding to $\alpha \operatorname{Ilb} \beta 3$, which triggers $\alpha \operatorname{IIb} \beta 3$-mediated outside-in signals and thus initiates cytoskeletal reorganization ${ }^{41,42}$. Platelets collected in $\mathrm{K}_{2}$-EDTA have the highest F-actin content under resting conditions in comparison to the other anticoagulants and show the lowest deformation. induced ultrastructural changes of the surface-bound canal system (narrowing and dilatation of the OCS) and an irreversible dissociation of the $\alpha \operatorname{IIb} \beta 3$ complexes ${ }^{43-45} 46$. It is possible that the high content of F-actin in non-stimulated and TRAP-6 stimulated platelets and the associated platelet deformation could be explained by the irreversible dissociation of the $\alpha I I b \beta 3$ complex and the associated cytoskeletal reorganization. p.E1841K platelets in ACD-A compared to $\mathrm{K}_{2}$-EDTA. The non-stimulated platelets in ACD-

24 A deform significantly more than those in $\mathrm{K}_{2}$-EDTA, even after TRAP-6 induced activation. 
1 of the human platelet cytoskeleton, while ACD-A, Na-Citrate, or r-Hirudin can be used.

2 These results may facilitate a comparison between different laboratories using shear-based

3 deformability cytometry such as RT-FDC to address fundamental questions of platelet

4 physiology and its relationship with biomechanical phenotype and may help to avoid artifacts

5 when these new technologies are applied to investigate patients with platelet disorders.

\section{Conclusions}

6 In summary, we can conclude that $\mathrm{K}_{2}$-EDTA and Li-Heparin influence the biomechanics of

7 platelets by decreasing the deformability and increasing actin polymerization of non-

8 stimulated human platelets. It is recommended for the examination of the human platelet

9 cytoskeleton to select an ex vivo anticoagulant such as ACD-A, Na-Citrate, or r-Hirudin and

10 not to exchange it if possible since comparability of the results cannot be guaranteed. With the

11 RT-FDC, we have a highly promising method to examine the platelet cytoskeleton in PRP,

12 which according to our study, provides very solid and fast results. 


\section{Material and Methods}

\section{Ethics}

1 The use of platelet-rich plasma (PRP) from healthy adult individuals and MYH9 patients was

2 approved by the ethics committee of the University Medicine Greifswald, Germany. All

3 participants gave written, informed consent

\section{Platelet Preparation}

4 The donors had not taken any medication in the previous ten days before blood collection.

5 Whole blood was collected by venipuncture in BD Vacutainer ${ }^{\circledR}$ Tubes containing acid citrate

6 dextrose solution A (ACD-A), 3.8\% buffered trisodium citrate (Na-Citrate), 102 I.U. Lithium-

7 Heparin (Li-Heparin), $1.8 \mathrm{mg} / \mathrm{mL}$ dipotassium ethylenediaminetetraacetic acid ( $\mathrm{K}_{2}$-EDTA),

8 or 171 ATU/mL recombinant hirudin (r-Hirudin) (REVASC, Canyon Pharmaceuticals, USA).

9 Whole blood was stored at room temperature for $15 \mathrm{~min}$ (at $45^{\circ}$ angle to the horizontal surface) and then centrifuged (120 x g for $20 \mathrm{~min}$ at room temperature). PRP was transferred to a new polypropylene tube and incubated for $15 \mathrm{~min}$ at $37^{\circ} \mathrm{C}$. All experimental measurements were performed within 3 hours of drawing the blood.

\section{Real-time fluorescence deformability cytometry (RT-FDC)}

13 The RT-FDC setup (AcCellerator, Zellmechanik Dresden, Germany) is built around an 14 inverted microscope (Axio Observer A1, Carl Zeiss AG, Germany) mounted with a Zeiss A15 Plan 100x NA 0.8 objective. The RT-FDC fluorescence module (Supplementary Fig. 1a) is 16 equipped with $488 \mathrm{~nm}, 561 \mathrm{~nm}, 640 \mathrm{~nm}$ excitation lasers, and emission is collected at the 17 following wavelengths: $500-550 \mathrm{~nm}, 570-616 \mathrm{~nm}, 663-737 \mathrm{~nm}$ on avalanche photodiodes 18 (Supplementary Fig.1a).

19 For functional mechanophenotyping of platelets based on molecular specificity in RT-FDC,

20 platelets in PRP were labeled with a mouse anti-human monoclonal antibody CD61-PE 21 (Beckman Coulter). Platelet activation was detected by direct immunofluorescence labeling of 22 alpha granule release marker CD62P (P-selectin) with mouse anti-human monoclonal 
1 antibody CD62P-AlexaFluor647 (Clone AK4, Cat.No. 304918, BioLegend, USA). and

2 activation associated conformational change in integrin $\alpha \operatorname{Ilb} \beta 3$ was detected with a mouse

3 anti-human monoclonal antibody PAC1-FITC (Clone PAC-1, Cat. No. 340507, B.D.

4 Biosciences, USA)and, respectively. PBS (Cat.No. P04-36500, PAN Biotech GmbH,

5 Germany) and TRAP-6 $(20 \mu \mathrm{M})$ (Haemochrom Diagnostica GmbH, Germany) were used as

6 vehicle control and platelet agonist, respectively. Incubations were performed at room

7 temperature for 10 minutes in the dark.

8 Deformability measurements were performed in a microfluidic chip with a constriction of 15

$9 \mu \mathrm{m} \times 15 \mu \mathrm{m}$ cross-section and a length of $300 \mu \mathrm{m}$ (Flic15, Zellmechanik Dresden, Germany)

10 (Supplementary Fig. 1b). Platelets in suspension are injected by a syringe pump (NemeSys,

11 Cetoni $\mathrm{GmbH}$, Germany), and cell deformation occurs due to the hydrodynamic pressure

12 gradient created by the surrounding fluid only. ${ }^{47}$

13 Based on cellular circularity, deformation is calculated on-the-fly using bright-field images

14 captured by a camera ${ }^{32}$ :

$$
\text { Deformation }=1-\frac{2 \sqrt{\pi A}}{P},
$$

where $A$ is the cross-sectional area of the cell and $P$ its perimeter.

RT-FDC measurements were carried out in buffer CellCarrier B (Zellmechanik Dresden,

Germany), which is composed of $0.6 \%$ (w/v) methylcellulose in PBS (without $\mathrm{Ca}^{2+}, \mathrm{Mg}^{2+}$ ).

Here, $50 \mu \mathrm{L}$ of immunofluorescently labeled PRP was suspended in $450 \mu \mathrm{L}$ CellCarrier B.

The PRP suspension was then driven through the microfluidic chip at flow rates of $0.006 \mu 1 / \mathrm{s}$, and the measurement was stopped after achieving 5000 single platelet count (hard-gate 150-

23 data was acquired using the ShapeIn software (Version 2.0, Zellmechanik Dresden,

24 Germany). Using the Shape-Out analysis software (https://github.com/ZELLMECHANIK-

25 DRESDEN/ShapeOut2/releases/tag/2.3.0 Version 2.3, Zellmechanik Dresden, Germany), 
1 kernel density estimation (KDE) plots of event density were generated, and statistical analysis

2 was performed to determine the median values for platelet deformation, their size and the

3 geometric mean of fluorescence (gMean) of the relevant functional variables. The range area

4 ratio was limited to $0-1.1$ and the cell size to $0-10 \mu \mathrm{m}$ for the analysis (Supplementary Fig. 2

5 and 3$)$.

\section{Flow cytometry}

6 Platelets were treated as described above for RT-DC. We used PerFix-nc Kit (Cat.No.

7 B31167; Beckman Coulter GmbH, Germany) and Phalloidin-Atto-647 (Atto-Tec GmbH,

8 Germany) to measure changes in total F-actin content in the platelets. Flow cytometry data

9 were processed using Flow $\mathrm{Jo}^{\mathrm{TM}}$ software for Windows, Version v10.6.2. (Becton, Dickinson

10 and Company, USA), and the gMean of the relevant variables was determined.

\section{Fluorescence Microscopy}

11 Platelets in PRP were incubated with PBS (vehicle control, non-stimulated)) or stimulated

with TRAP-6 for 10min followed by fixation in 2\% paraformaldehyde (Morphisto, Germany)

for $15 \mathrm{~min}$. Fixed platelets were transferred into a Shandon ${ }^{\mathrm{TM}}$ Single Cytofunnel $^{\mathrm{TM}}$ (Thermo

Fisher, USA) and were centrifuged on a microscope slide for $5 \mathrm{~min}$ at 700rpm (Cytospin

ROTOFIX 32 A, Hettich, Germany), washed thrice with PBS (5 min intervals). Platelet were

permeabilized in $0.5 \%$ saponin with $0.2 \%$ bovine serum albumin (BSA) (Cat. No. 11924.03,

SERVA Electrophoresis GmbH, Germany ) for $25 \mathrm{~min}$, and followed by blocking for $30 \mathrm{~min}$ platelets were incubated with 1:500 dilution of mouse monoclonal anti- $\alpha$-Tubulin IgG (Clone DM1A, Cat.No. T9026, Sigma Aldrich GmbH, Germany) primary antibody diluted in $0.5 \%$ saponin with $0.2 \%$ BSA in PBS for 16 hours at $4^{\circ} \mathrm{C}$ followed by three washing steps in PBS for $5 \mathrm{~min}$ each. Afterward, platelets were incubated with 1:750 dilution of goat polyclonal anti-mouse AF488 IgG prepared in $0.5 \%$ saponin with $0.2 \%$ BSA in PBS for 60

24 minutes in the dark at room temperature, followed by three washing steps with PBS for 5 min 
1 each. F-actin was labeled with 20 pM Phalloidin Atto 647N (Cat. No. AD 647N-81, Atto-Tec

$2 \mathrm{GmbH}$, Germany) for 60 minutes, followed by three washes in PBS for 5 minutes each. Slides

3 were covered by a permanent mounting medium Roti®-Mount FluorCare (Cat. No. HP19.1,

4 Karl-Roth GmbH, Germany). Fluorescence microscopy was performed on a Leica SP5

5 confocal laser scanning microscope (Leica Microsystems, Wetzlar, Germany) equipped with

6 HCX PL APO lambda blue 40x/1.25 OIL UV objective. For image acquisition, AF488 and

7 ATTO647 were excited by argon $(488 \mathrm{~nm})$ and helium-neon $(\mathrm{HeNe})(633 \mathrm{~nm})$ laser lines

8 selected with an acousto-optic tunable filter (AOTF), and fluorescence emission was collected

9 between 505-515 $\mathrm{nm}$ and 640-655 nm respectively on hybrid detectors (HyD). Assessment of

10 F-actin distribution and organization of marginal band $\alpha$-tubulin staining was performed by

11 measuring cross-sectional line profile $(5 \mu \mathrm{m}$ length and $1 \mu \mathrm{m}$ width) of non-saturated

12 grayscale fluorescence intensities (pixel values) of immunofluorescent probes across

13 individual platelets in confocal images using Leica Application Suite X (Version 3.7.1, Leica

14 Microsystems, Wetzlar, Germany). For data plotting, GraphPad Prism version 8.0.0 for

15 Windows (GraphPad Software, San Diego, California USA) was used.

\section{Statistical plots and analysis of RT-FDC data}

16 Statistical plots showing parameters of the platelet population were prepared with ShapeOut software (https://github.com/ZELLMECHANIK-DRESDEN/ShapeOut2/releases/tag/2.3.0

Version

2.3

Zellmechanik

Dresden),

PlotsOfDifferences (https://gabrifc.shinyapps.io/raincloudplots/). ${ }^{48,49}$ Statistical assessment was performed using the mixed-effects model (restricted maximum likelihood, REML) followed by Tukey's multiple comparisons test, with single pooled variance using GraphPad Prism version 8.0.0

23 for Windows (GraphPad Software, San Diego, California USA). $P<0.05$ was considered 24 significant. 


\section{Data availability}

1 Source data and accompanying software for analysis of RT-FDC data are available in a

2 citable repository and can be accessed by requesting the corresponding author(s).

\section{Acknowledgments}

3 O.O gratefully acknowledges funding from the German Ministry of Education and Research

4 (BMBF) within the project 03Z22CN11 (ZIK grant) and the German Center for

5 Cardiovascular Research within the project 81X3400107 (Postdoc start-up grant). This work

6 was supported by the Deutsche Forschungsgemeinschaft project number 374031971-CRC/TR

7240 project A06 to M.B., O.O. and R.P.

\section{Author contributions}

8 L.S., A.G.,M.B.,O.O. and R.P. designed the study. L.S. performed all RT-FDC experiments.

9 J.W. and L.L. performed flow cytometry. L.S. and R.P. performed CLSM experiments. L.S., 10 and R.P. analyzed the data and prepared figures. L.S. wrote the manuscript. A.G. provided 11 access to MYH9 patient platelets. A.G., M.B., O.O. and R.P. contributed to writing the 12 manuscript. All authors contributed to the critical revision of the manuscript. .M.B., O.O. and 13 R.P. obtained funding.

\section{Conflict of interest}

14 O.O. is co-founder and shareholder of Zellmechanik Dresden $\mathrm{GmbH}$, distributing real-time 15 deformability cytometry. 


\section{References}

1. Machlus, K.R. \& Italiano, J.E., Jr. The incredible journey: From megakaryocyte development to platelet formation. J Cell Biol 201, 785-796 (2013).

2. Jurk, K. \& Kehrel, B.E. Platelets: physiology and biochemistry. Semin Thromb Hemost 31, 381-392 (2005).

3. Koupenova, M., Clancy, L., Corkrey, H.A. \& Freedman, J.E. Circulating Platelets as Mediators of Immunity, Inflammation, and Thrombosis. Circ Res 122, 337-351 (2018).

4. Gaertner, F. \& Massberg, S. Blood coagulation in immunothrombosis-At the frontline of intravascular immunity. Semin Immunol 28, 561-569 (2016).

5. Hansen, C.E., Qiu, Y., McCarty, O.J.T. \& Lam, W.A. Platelet Mechanotransduction. Annu Rev Biomed Eng 20, 253-275 (2018).

6. Zhang, Y., et al. Platelet integrins exhibit anisotropic mechanosensing and harness piconewton forces to mediate platelet aggregation. Proc Natl Acad Sci U S A 115, 325-330 (2018).

7. Qiu, Y., Myers, D.R. \& Lam, W.A. The biophysics and mechanics of blood from a materials perspective. Nat Rev Mater 4, 294-311 (2019).

8. Qiu, Y., Ciciliano, J., Myers, D.R., Tran, R. \& Lam, W.A. Platelets and physics: How platelets "feel" and respond to their mechanical microenvironment. Blood Rev 29, 377-386 (2015).

9. Kim, O.V., Litvinov, R.I., Alber, M.S. \& Weisel, J.W. Quantitative structural mechanobiology of platelet-driven blood clot contraction. Nat Commun 8, 1274 (2017).

10. Lam, W.A., et al. Mechanics and contraction dynamics of single platelets and implications for clot stiffening. Nat Mater 10, 61-66 (2011).

11. Gaertner, F., et al. Migrating Platelets Are Mechano-scavengers that Collect and Bundle Bacteria. Cell 171, 1368-1382 e1323 (2017).

12. Palankar, R., et al. Platelets kill bacteria by bridging innate and adaptive immunity via platelet factor 4 and FcgammaRIIA. J Thromb Haemost 16, 1187-1197 (2018).

13. Nicolai, L., et al. Vascular surveillance by haptotactic blood platelets in inflammation and infection. Nat Commun 11, 5778 (2020).

14. Myers, D.R., et al. Single-platelet nanomechanics measured by high-throughput cytometry. Nat Mater 16, 230-235 (2017).

15. White, J.G., Burris, S.M., Tukey, D., Smith, C., 2nd \& Clawson, C.C. Micropipette aspiration of human platelets: influence of microtubules and actin filaments on deformability. Blood 64, 210-214 (1984).

16. Burris, S.M., Smith, C.M., 2nd, Tukey, D.T., Clawson, C.C. \& White, J.G. Micropipette aspiration of human platelets after exposure to aggregating agents. Arteriosclerosis 6, 321-325 (1986).

17. Haga, J.H., Beaudoin, A.J., White, J.G. \& Strony, J. Quantification of the passive mechanical properties of the resting platelet. Ann Biomed Eng 26, 268-277 (1998). 
18. Radmacher, M., Fritz, M., Kacher, C.M., Cleveland, J.P. \& Hansma, P.K. Measuring the viscoelastic properties of human platelets with the atomic force microscope. Biophys J 70, 556-567 (1996).

19. Sorrentino, S., Studt, J.D., Horev, M.B., Medalia, O. \& Sapra, K.T. Toward correlating structure and mechanics of platelets. Cell Adh Migr 10, 568-575 (2016).

20. Nguyen, T.H., et al. Rupture Forces among Human Blood Platelets at different Degrees of Activation. Sci Rep 6, 25402 (2016).

21. Rheinlaender, J., et al. Imaging the elastic modulus of human platelets during thrombin-induced activation using scanning ion conductance microscopy. Thromb Haemost 113, 305-311 (2015).

22. Seifert, J., Rheinlaender, J., Lang, F., Gawaz, M. \& Schaffer, T.E. Thrombin-induced cytoskeleton dynamics in spread human platelets observed with fast scanning ion conductance microscopy. Sci Rep 7, 4810 (2017).

23. Schwarz Henriques, S., Sandmann, R., Strate, A. \& Koster, S. Force field evolution during human blood platelet activation. J Cell Sci 125, 3914-3920 (2012).

24. Hanke, J., Probst, D., Zemel, A., Schwarz, U.S. \& Koster, S. Dynamics of force generation by spreading platelets. Soft Matter 14, 6571-6581 (2018).

25. Liang, X.M., Han, S.J., Reems, J.A., Gao, D. \& Sniadecki, N.J. Platelet retraction force measurements using flexible post force sensors. Lab Chip 10, 991-998 (2010).

26. Feghhi, S., et al. Glycoprotein Ib-IX-V Complex Transmits Cytoskeletal Forces That Enhance Platelet Adhesion. Biophys J 111, 601-608 (2016).

27. Lickert, S., et al. Platelets exploit fibrillar adhesions to assemble fibronectin matrix revealing new force-regulated thrombus remodeling mechanisms. bioRxiv, 2020.2004.2020.050708 (2020).

28. Sachs, L., Denker, C., Greinacher, A. \& Palankar, R. Quantifying single-platelet biomechanics: An outsider's guide to biophysical methods and recent advances. Res Pract Thromb Haemost 4, 386-401 (2020).

29. Penington, D.G., Streatfield, K. \& Roxburgh, A.E. Megakaryocytes and the heterogeneity of circulating platelets. Br J Haematol 34, 639-653 (1976).

30. Corash, L., Tan, H. \& Gralnick, H.R. Heterogeneity of human whole blood platelet subpopulations. I. Relationship between buoyant density, cell volume, and ultrastructure. Blood 49, 71-87 (1977).

31. Radziwon-Balicka, A., et al. Differential eNOS-signalling by platelet subpopulations regulates adhesion and aggregation. Cardiovasc Res 113, 1719-1731 (2017).

32. Otto, O., et al. Real-time deformability cytometry: on-the-fly cell mechanical phenotyping. Nat Methods 12, 199-202, 194 p following 202 (2015).

33. Rosendahl, P., et al. Real-time fluorescence and deformability cytometry. Nat Methods 15, 355-358 (2018).

34. Herbig, M., et al. Image-based cell sorting using artificial intelligence. 17 (2020).

35. Nawaz, A.A., et al. Intelligent image-based deformation-assisted cell sorting with molecular specificity. Nat Methods 17, 595-599 (2020). 
36. Jacobi, A., et al. Analysis of Biomechanical Properties of Hematopoietic Stem and Progenitor Cells Using Real-Time Fluorescence and Deformability Cytometry. Methods Mol Biol 2017, 135-148 (2019).

37. Luhr, J.J., et al. Maturation of Monocyte-Derived DCs Leads to Increased Cellular Stiffness, Higher Membrane Fluidity, and Changed Lipid Composition. Front Immunol 11, 590121 (2020).

38. Guck, J. Some thoughts on the future of cell mechanics. Biophys Rev 11, 667-670 (2019).

39. Shattil, S.J., Motulsky, H.J., Insel, P.A., Flaherty, L. \& Brass, L.F. Expression of fibrinogen receptors during activation and subsequent desensitization of human platelets by epinephrine. Blood 68, 1224-1231 (1986).

40. Canobbio, I., et al. Altered cytoskeleton organization in platelets from patients with MYH9-related disease. J Thromb Haemost 3, 1026-1035 (2005).

41. Gao, C., et al. Heparin promotes platelet responsiveness by potentiating alphaIIbbeta3-mediated outside-in signaling. Blood 117, 4946-4952 (2011).

42. Sobel, M., et al. Heparin modulates integrin function in human platelets. J Vasc Surg 33, 587-594 (2001).

43. Gachet, C., et al. Alpha IIb beta 3 integrin dissociation induced by EDTA results in morphological changes of the platelet surface-connected canalicular system with differential location of the two separate subunits. J Cell Biol 120, 1021-1030 (1993).

44. Golanski, J., Pietrucha, T., Baj, Z., Greger, J. \& Watala, C. Molecular insights into the anticoagulant-induced spontaneous activation of platelets in whole blood-various anticoagulants are not equal. Thromb Res 83, 199-216 (1996).

45. White, J.G. EDTA-induced changes in platelet structure and function: clot retraction. Platelets 11, 49-55 (2000).

46. Ma, Y. \& Wong, K. Reassociation and translocation of glycoprotein IIB-IIIA in EDTA-treated human platelets. Platelets 18, 451-459 (2007).

47. Mietke, A., et al. Extracting Cell Stiffness from Real-Time Deformability Cytometry: Theory and Experiment. Biophys J 109, 2023-2036 (2015).

48. Goedhart, J. PlotsOfDifferences - a web app for the quantitative comparison of unpaired data. bioRxiv, 578575 (2019).

49. Allen, M., Poggiali, D., Whitaker, K., Marshall, T. \& Kievit, R. Raincloud plots: a multi-platform tool for robust data visualization [version 1; peer review: 2 approved]. Wellcome Open Research 4(2019). 